\title{
Erratum: Gravity-mediated scalar Dark Matter in warped extra-dimensions
}

\section{Miguel G. Folgado, Andrea Donini and Nuria Rius}

Departamento de Física Teórica and IFIC, Universidad de Valencia-CSIC,

C/ Catedrático José Beltrán, 2, E-46980 Paterna, Spain

E-mail: migarfol@ific.uv.es, donini@ific.uv.es, nuria.rius@ific.uv.es

ERRATUM TO: JHEP01(2020)161

ArXIV EPRINT: 1907.04340 
After this article was published, we became aware of refs. [1, 2], where it was shown that the fast growth of the scalar DM annihilation cross section into KK-gravitons for large DM mass $\left(m_{\mathrm{DM}} \gg m_{G_{n}}\right)$ is in fact an artifact of not summing over the full tower of KK-gravitons of the 5-dimensional theory. When one adds coherently to our result for the cross-section $S S \rightarrow G_{n} G_{m}$ in eq. (D.9) the contributions from the triple KK-graviton $\left(G_{k} G_{n} G_{m}\right)$ and the radion and two KK-gravitons $\left(r G_{n} G_{m}\right)$ vertices and, moreover, one includes the full tower of virtual KK-gravitons in the $s$-channel, a cancellation of the most divergent terms takes place (those coming from the longitudinal polarization of the spin-2 KK-gravitons). The resulting cross-section has the expected behaviour $\sigma_{g}\left(S S \rightarrow G_{n} G_{m}\right) \propto m_{\mathrm{DM}}^{2} / \Lambda^{4}$ for $m_{\mathrm{DM}} \gg m_{G_{n, m}}$, in the small DM relative velocity approximation.

In ref. [2], the authors reconsider gravity-mediated scalar Dark Matter within the Randall-Sundrum scenario, studied in this paper, and they find that once the summation over all the KK virtual gravitons is performed, the DM annihilation cross-section into KKgravitons is indeed subleading with respect to the annihilation into SM particles. This changes drastically our previous results, summarized in figure 10 of the paper. We refer the readers to refs. $[1,2]$ for the technical details, and here we just have updated our plots using the corrected DM annihilation cross-section, now dominated by the SM final states. Notice that we restrict ourselves to the case in which the radion plays a role, since we find that otherwise it is not possible to reproduce the Dark Matter relic abundance in any point allowed by current LHC bounds and theoretical constraints.

In particular, figure 1 (left panel) shows the new values of $\Lambda$ needed to obtain the correct relic abundance for $m_{r}=1 \mathrm{TeV}$. The resonance of the radion appears at $m_{\mathrm{DM}}=m_{r} / 2$. As a consequence of the radion resonance, the value of $\Lambda$ increases at this point, in order to achieve the thermal cross-section. An allowed region corresponding to a resonant radionexchange channel is always present. However, it relies on a high degree of fine-tuning between the DM and radion masses, unjustified in the absence of a specific model that relates the two particles. For this reason, in our paper we did not highlight this possibility. We show this particular value of $m_{r}$ here in order to compare more easily with the results of ref. [2]. Figure 1 (right panel), on the other hand, shows the region in the $\left(m_{\mathrm{DM}}, m_{G_{1}}\right)$ plane for which the observed DM relic abundance is obtained, together with experimental bounds from DM direct detection and LHC resonant KK-graviton searches, as well as theoretical constraints, namely the unitarity limit and the region where the perturbativity of the effective theory is doubtful. In agreement with ref. [2], we find that it is possible to reproduce the DM energy density via freeze-out and comply with all the constraints near the radion resonance, $m_{\mathrm{DM}} \simeq m_{r} / 2$ (we have taken $m_{r}=1 \mathrm{TeV}$ ). In ref. [2], a second small region that escapes LHC bounds due to the kinematic limit $\left(m_{G_{1}}>5 \mathrm{TeV}, m_{\mathrm{DM}} \sim 2 \mathrm{TeV}\right)$ exists. This region, however, corresponds to $m_{G_{1}}>\Lambda$, which we disregard. Notice that the perturbative limit imposed in ref. [2], $\Gamma_{1}<m_{G_{1}} / 2$ (being $\Gamma_{1}$ the first KK-graviton decay width), is a bit looser than ours and it is satisfied in such region. On the other hand, we find an allowed region for $m_{G_{1}}>5 \mathrm{TeV}, m_{\mathrm{DM}} \sim 10 \mathrm{TeV}$, close to the unitarity limit, that in ref. [2] is excluded by their stronger unitarity bound. In both regions the reliability of the perturbative calculation is a concern. 

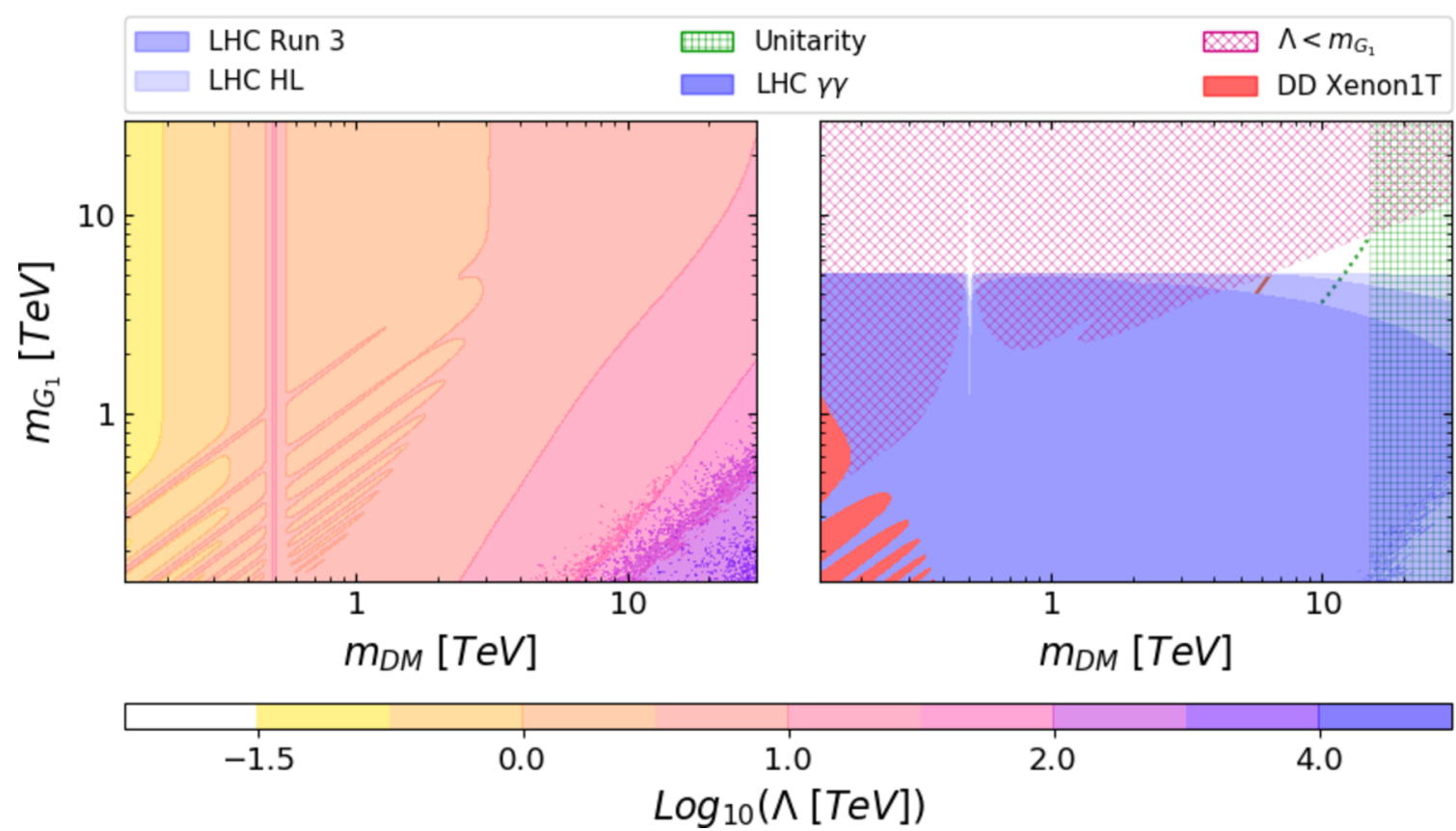

Figure 1. Left panel: values of $\Lambda$ for which the correct DM relic abundance is obtained in the plane $\left(m_{\mathrm{DM}}, m_{G_{1}}\right)$ with a radion mass $m_{r}=1 \mathrm{TeV}$. The required $\Lambda$ ranges from $300 \mathrm{GeV}$ to $10^{5} \mathrm{TeV}$, as shown by the color legend. This figure updates figure 5 of our paper, albeit with a choice of the radion mass large enough to give the vertical line around $m_{\mathrm{DM}} \sim 500 \mathrm{GeV}$, corresponding to a resonant exchange of the radion in $s$-channel. Right panel: region of the $\left(m_{\mathrm{DM}}, m_{G_{1}}\right)$ plane for which $\langle\sigma v\rangle=\left\langle\sigma_{\mathrm{FO}} v\right\rangle$ with radion mass $m_{r}=1 \mathrm{TeV}$. The red-meshed area is the region for which the low-energy RS effective theory is untrustable, as $\Lambda<m_{G_{1}}$; the wiggled pink area in the lower left corner is the region excluded by Direct Dark Matter searches; the blue area is excluded by resonant KK-graviton searches at the $\mathrm{LHC}$ with $36 \mathrm{fb}^{-1}$ at $\sqrt{s}=13 \mathrm{TeV}$ (dark blue), at the end of the Run III with expected $\sim 300 \mathrm{fb}^{-1}$ (blue) and at the HL-LHC with expected $\sim 3000 \mathrm{fb}^{-1}$ (light blue); eventually, the green-meshed area on the right is the region where the theoretical unitarity constraints are not fulfilled. The allowed region is represented by the white area. Notice that a tiny allowed region, mostly independent of $m_{G_{1}}$, is always present for $m_{\mathrm{DM}} \simeq m_{r} / 2$. As a reference, in the allowed region we have drawn two lines corresponding to the value of $\Lambda$ needed to get $\langle\sigma v\rangle=\left\langle\sigma_{\mathrm{FO}} v\right\rangle$ : the orange-dashed line stands for $\Lambda=5 \mathrm{TeV}$, the green-dotted line for $\Lambda=8 \mathrm{TeV}$.

Finally, in appendix C. 1 of our paper we said that the decay width of the KK-graviton into KK-gravitons is negligible with respect to decay into SM particles due to the fact that the triple KK-graviton vertex is proportional to $\Lambda^{-3}$. This is clearly wrong, as in refs. [1, 2$]$ it was correctly shown that the triple KK-gravitons vertex is indeed proportional to $\Lambda^{-1}$ and, therefore, not suppressed with respect to the $G_{n} \rightarrow$ SM SM vertex. However, we have found that the decay width of the KK-gravitons into two KK-gravitons, one KK-graviton and one radion, or two radions is indeed negligible with respect to the decay width into SM particles and, therefore our conclusion was correct.

Notice that the surviving region of the parameter space could only be accessed by future accelerators such as the FCC-hh or HE-LHC at CERN, with a c.o.m. energy in the range of several tens of $\mathrm{TeV}[3,4]$. 
Open Access. This article is distributed under the terms of the Creative Commons Attribution License (CC-BY 4.0), which permits any use, distribution and reproduction in any medium, provided the original author(s) and source are credited.

\section{References}

[1] A. de Giorgi and S. Vogl, Unitarity in KK-graviton production: a case study in warped extra-dimensions, JHEP 04 (2021) 143 [arXiv: 2012.09672] [INSPIRE].

[2] A. de Giorgi and S. Vogl, Dark matter interacting via a massive spin-2 mediator in warped extra-dimensions, JHEP 11 (2021) 036 [arXiv:2105.06794] [INSPIRE].

[3] FCC collaboration, FCC physics opportunities. Future Circular Collider conceptual design report volume 1, Eur. Phys. J. C $\mathbf{7 9}$ (2019) 474 [InSPIRE].

[4] FCC collaboration, FCC-ee: the lepton collider. Future Circular Collider conceptual design report volume 2, Eur. Phys. J. ST 228 (2019) 261 [INSPIRE]. 